\title{
An Improved Unordered Pair Bat Algorithm for Solving the Symmetrical Traveling Salesman Problem
}

\author{
Zhang Nan, Lv Zhimin, Qiao Shen *, Li Ting ${ }^{1}$
}

\begin{abstract}
Bat algorithm is an effective swarm intelligence optimization algorithm which is widely used to solve continuous optimization problems. But it still has some limitations in search process and can't solve discrete optimization problems directly. Therefore, this paper introduces an unordered pair and proposes an unordered pair bat algorithm (UPBA) to make it more suitable for solving symmetric discrete traveling salesman problems. To verify the effectiveness of this method, the algorithm has been tested on 23 symmetric benchmarks and compared its performance with other algorithms. The results have shown that the proposed UPBA outperforms all the other alternatives significantly in most cases.
\end{abstract}

Keywords: symmetrical traveling salesman problem, discrete bat algorithm, unordered pair bat algorithm.

\section{Introduction}

Combinatorial optimization problem is an important academic field and an important branch of modern operational research. Currently, the typical combinatorial optimization problems include traveling salesman problem (TSP) [15], vehicle routing problem (VRP) [28], bin packing problem[26], 0-1 knapsack problem[25], clustering problem[5], and so on. Most of the combinatorial optimization problems mentioned above are NP-hard, and the solutions are often difficult to find. In view of this, various of appropriate methods can be found in the literature to solve such problems effectively. It can be said that the most successful techniques are exact algorithm, approximation algorithm, heuristics algorithm, and metaheuristics algorithm.

An exact algorithm can find the optimal solution to the problem. When the scale of the problem is small, the exact algorithm can get the optimal solution in an acceptable time. The commonly used algorithms are branch and bound method, cut plane method, dynamic programming method. Approximate algorithms are used to solve optimization problems by

*Collaborative Innovation Center of steel technology, University of Science and Technology Beijing, No.30, Xueyuan Road, Haidian District, Beijing, China.

Corresponding author: Lv Zhimin. Email: YeahDS01@126.com.

${ }^{1}$ Beijing Jinghang Research Institute of Computing and Communication, Beijing, China 
approximate methods, which are usually related to NP-hard problems. Local search algorithm, relaxation algorithm and dynamic programming method can be used to construct approximate algorithms. Heuristic algorithm is an algorithm based on intuition or experience. It gives a feasible solution of the combinatorial optimization problem to be solved under an acceptable cost. Heuristic algorithms include local search algorithm, relaxation method, solution space reduction algorithm, etc. Metaheuristic algorithm mainly refers to a kind of general heuristic algorithm. It improves the heuristic algorithm and is the product of the combination of random algorithm and local search algorithm. Metaheuristic algorithm mainly includes tabu search algorithm (TS) [13], simulated annealing algorithm(SA) [29], genetic algorithm (GA) [31], ant colony algorithm [7], particle swarm optimization algorithm (PSO) [8], cuckoo search algorithm (CS) [11], bat algorithm (BA) [32], artificial neural network and so on. Most of the above algorithms belong to swarm intelligence algorithms. Swarm intelligence algorithm is a random search algorithm using swarm. By defining group behavior and individual behavior, the swarm has population evolution diversity and behavior orientation. These properties can be used to approximately solve some optimization problems that are difficult to solve directly. With the development of the metaheuristic and swarm intelligence algorithms in the past few decades, these algorithms are widely used to solve combinatorial optimization problems.

Therefore, this paper mainly uses a metaheuristic algorithm, called bat algorithm, to solve a typical combinatorial optimization problem. Here, the TSP is used for the experimentation. It is one of the typical NP-hard combinatorial optimization problems applied in many fields $[6,9]$ and is difficult to solve. How to solve the TSP quickly and accurately has been the focus of scientific researchers. Bat algorithm (BA) was proposed by Yang in 2010 [32], and it simulates the process that bats use sonar to find prey and avoid obstacles in nature. The algorithm is used primarily for solving different optimization problems in most cases. With the development of the algorithm, it is also used to solve discrete problems and practical engineering problems in recent years. However, the BA is still in its early stage for solving the routing problems such as TSP and VRP. Therefore, in recent years, many scholars have been attracted to this kind of problem, which is also the focus of this paper.

To sum up, the main research of this paper is to propose an improved discrete BA, which is called unordered pair bat algorithm, to solve the symmetric TSP. In the process of algorithm improvement, an unordered pair is proposed to improve the updating of velocity of bats. In addition, a new definition of pulse emission rate and loudness is proposed to implement the algorithm more efficiently.

In order to verify the UPBA is an effective method for TSP, the improved algorithm has been tested on 23 symmetric benchmarks and compared its performance with the ones obtained by bat algorithm without improved parameters, and with the ones obtained by other algorithms: genetic simulated annealing ant colony system with particle swarm optimization techniques algorithm (GSA-ACS-PSOT) [4], Real-valued antibody network algorithm (RABNET-TSP) [20] and the Improved BA[22].

The remaining part of this paper is organized as follows: Section 2 introduces a literature review of BA and TSP. Section 3 focuses on the details of basic BA. Section 4 briefly describes the TSP model. In section 5, an improved unordered pair bat algorithm is proposed according to the symmetric TSP. Section 6 presents a set of experimentation of symmetric TSP benchmarks from the TSPLIB library. Finally, summaries and future work are explained in Section 7. 


\section{Related work}

Swarm intelligence algorithm has been widely used to solve optimization problems in recent years, such as PSO, ACO, BA, artificial fish swarm, bee colony optimization, and so on. Most of these algorithms are inspired by natural groups' laws, and use the individual interaction information to form the regular motion.

Bat algorithm is one of the metaheuristic search algorithms based on swarm intelligence. The algorithm is based on iterative optimization techniques. The initial random solution is searched iteratively and the local solution is generated by a random flight. Compared with other algorithms, BA does not have many parameters to be adjusted, and it is more accurate and effective.

In the initial period, BA was applied primarily for solving large-scale continuous problems. Since differential-evolution supports differential mutation, crossover and selection, Fister et al. [10] proposed to use differential-evolution strategies to solve higherdimensional continuous problems. He et al. [14] proposed simulated annealing and Gaussian perturbations into the standard bat algorithm. These methods are used to improve the search performance, and the results show that the proposed algorithm can improve global convergence. Because chaos is easier to jump out of local optimum and improve global convergence, it is extended to solve optimization problems. Afrabandpey et al. [2] introduced chaotic sequences to initialize the algorithm parameters of bats and improved their ability to jump out of the local optimum. Gandomi and yang [12] used different chaotic systems to replace the parameters in BA to increase its global search mobility. Zhou Y et al. [33] introduced a cloud model to depict the qualitative concept: "bats approach their prey.", and added random Levy flight in the algorithm to improve the ability of escape the local optimum. These papers solve continuous problems by replacing the parameters in BA and changing the solution strategy, which can speed up the convergence and find feasible solutions.

Most of the practical optimization problems are considered discrete problems. Currently, the basic bat algorithm has not been able to solve discrete optimization problems directly. Therefore, many scholars have researched the solution to adaptive BA of optimization problems. Marichelvam et al. $[18,19]$ introduced the smallest position value (SPV) rule to transform the continuous position into discrete process sequences in BA for solving mixed flow shop scheduling problem. Abdel-Raouf et al. [1] proposed an improved chaotic bat algorithm for solving integer programming problems. They use a chaotic map to replace the random number $\beta$, and change the velocity function. Mirjalili et al. [21] constructed a binary bat algorithm by constructing the $V$ shape transfer function, and conducted a comparative study with binary PSO and GA. The results proved that the proposed algorithm was able to outperform others significantly.

As a standard test problem, TSP is mainly used in performance analysis of combinatorial optimization problems. A lot of work had been done to analyze discrete TSP problems in recent years.

Pedro O et al. [24]proposed a simple, but efficient tabu search approach to solve the Prize Collecting Traveling Salesman Problem. Lin Y et al. [16]combined tabu search with simulated annealing algorithm to solve the TSP. Fully considering the characteristics of the hybrid algorithm, they develop a dynamic neighborhood structure and adaptive parameters. The experimental results demonstrate the improved accuracy and efficiency of the proposed algorithm. Wang $\mathrm{J}$ et al. [30] provided a multi-offspring genetic algorithm (MO-GA) and 
illustrated its application in the TSP. The MO-GA increased the probability of producing excellent individuals, and also made the population more competitive. Ouaarab A et al. [23] extended and improved Cuckoo Search (CS) by reconstructing its population and introducing a new category of cuckoos to solve TSP. Mahi M et al. [17] proposed a new hybrid method to optimize parameters that affect performance of the ACO algorithm using Particle Swarm Optimization (PSO). And the hybrid method was compared to the performance of some wellknown algorithms.

In recent years, BA has been successfully and widely used to solve TSP problems due to its advantages. Al-Sorori et al. [3] constructed an improved hybrid variant of discrete bat algorithm to solve traveling salesman problem. Saji and Riffi [27] proposed a discrete BA algorithm to solve the symmetric TSP by using the 2-exchange crossover mechanism, and the results revealed the discrete algorithm performs all compared algorithms. Osaba et al. [22] adapted $v_{i}^{t}$ using the well-known Hamming Distance to present a discrete bat algorithm to solve the well-known symmetric and asymmetric TSP, and compared its performance in 37 instances. These papers solve discrete problems by changing the velocity and position of individual, and these methods have been proved to be effective.

However, BA still has some limitations including stagnation in the early stages, loss of exploration as the iterations flow, premature convergence, etc. Therefore, this paper introduces the concept of unordered pair to update the speed and position of bats, and proposes an unordered pair bat algorithm (UPBA) to make it more suitable for solving symmetric discrete traveling salesman problems.

\section{Bat algorithm}

The BA is a random search algorithm that simulates the sonar system of bats to detect prey, avoid obstacles and locate their roosting crevices in the dark. The implementation process is described as follows [32]:

Step1: Population initialization. The bats generate a set of initial solutions randomly in $\boldsymbol{D}$-dimensional search space. The parameters are defined as follows: the number of bats $i$, the iteration number $t$, the loudness $A_{i}$, the rate of pulse emission $r_{i}$, loudness attenuation coefficient $\alpha$, emission enhancement coefficient $\gamma$, where the $\alpha$ and $\gamma$ are constants. And each bat is randomly assigned a frequency $f_{i} \in\left[f_{\text {min }}, f_{\text {max }}\right]$.

Step2: Randomly initialize the bat position $x_{i}$, find the current global best location (solution) $x_{*}$.

Step3: The movement of each bat is given by updating their velocities $v_{i}^{t}$ and positions $x_{i}^{t}$ using Eqs. 1 to 3 as follows:

$$
\begin{gathered}
f_{i}=f_{\text {min }}+\left(f_{\text {max }}-f_{\text {min }}\right) * \beta \\
v_{i}^{t}=v_{i}^{t-1}+\left(x_{i}^{t-1}-x_{*}\right) * f_{i} \\
x_{i}^{t}=x_{i}^{t-1}+v_{i}^{t}
\end{gathered}
$$

Where $\beta \in[0,1]$ denotes a uniformly distributed random number, $x_{*}$ denotes the current global best location, which is located after comparing all the bats.

Step4: A random number is generated, if the random number $r$ and $>r_{i}$, Select a solution among the best ones, and generate a local solution around the selected best solution, it can be represented by:

$$
x_{\text {new }}=x_{\text {old }}+\varepsilon A_{i}^{t}
$$


where $\varepsilon \in[0,1]$ denotes a random number, and $A_{i}^{t}$ is the average loudness of all the bats in the current generation.

Step5: A random number is generated, if the random number $r a n d<A_{i}^{t}$ and $f\left(x_{i}\right)<$ $f\left(x_{*}\right)$, the above solution is accepted and $A_{i}$ and $r_{i}$ are updated by Eqs.5 and 6 as follows:

$$
\begin{gathered}
A_{i}^{t+1}=\alpha A_{i}^{t} \\
r_{i}^{t+1}=R_{0}\left[1-e^{-\gamma t}\right]
\end{gathered}
$$

where $R_{0}$ is the initial emission rate.

Step6: Rank the bats and find the current best $x_{*}$.

Step7: Repeat Step2 Step6 until the maximum iterations are achieved.

Step8: Output the best solution, post-process the results and visualization.

In conclusion, the pseudo-code of the basic bat algorithm is shown in Algorithm1.

Algorithm1 Pseudo-code of the basic BA

1 Define the objective function $\boldsymbol{f}(\boldsymbol{x})$;

2 Initialize the bat population $X=x_{1}, x_{2}, \ldots \ldots, x_{n}$;

3 for each bat $\boldsymbol{x}_{\boldsymbol{i}}$ in the population

4 Initialize the rate of pulse emission $\boldsymbol{r}_{\boldsymbol{i}}$, velocity $\boldsymbol{v}_{\boldsymbol{i}}$ and loudness $\boldsymbol{A}_{\boldsymbol{i}}$;

5 Define the pulse frequency $\boldsymbol{f}_{\boldsymbol{i}}$ at $\boldsymbol{x}_{\boldsymbol{i}}$;

6 end for

7 While ( $\mathrm{t}<$ Max number of iterations)

8 Generation new solutions through Eqs.1, 2 and 3;

9 if $\operatorname{rand}>\boldsymbol{r}_{\boldsymbol{i}}$

10 Select a solution among the best ones;

11 Generate a local solution around the selected best solution;

12 end if

13 generation a new solution by flying randomly;

14 if rand $<\boldsymbol{A}_{\boldsymbol{i}}$ and $\boldsymbol{f}\left(\boldsymbol{x}_{\boldsymbol{i}}\right)<\boldsymbol{f}\left(\boldsymbol{x}_{*}\right)$

15 Accept the new solution;

16 Increase $\boldsymbol{r}_{\boldsymbol{i}}$ and reduce $\boldsymbol{A}_{\boldsymbol{i}}$ through Eqs.5 and 6;

17 end if

18 Rank the bats and find the current best $\boldsymbol{x}_{*}$

19 end while

20 Post-process results and visualization;

\section{Traveling salesman problem}

The traveling salesman problem (TSP) is a classic NP-hard combinatorial optimization problem in computer science and routing selection. It can be described as a problem to find the shortest closed tour which visits all the cities in given coordinates. In other words, its mathematical description can be abstracted as: given a set of $n$ cities, named $\left(C_{1}, C_{2}, \ldots, C_{n}\right)$ and the Euclidean distance $d$ of any two cities is known in advance. The Euclidean distance of cities with coordinate $\left(x_{1}, y_{1}\right)$ and $\left(x_{2}, y_{2}\right)$ is calculated by:

$$
d=\sqrt{\left(x_{1}-x_{2}\right)^{2}+\left(y_{1}-y_{2}\right)^{2}}
$$


For a given order $T=\left(t_{1}, t_{2}, \ldots t_{n}\right)$, and record $t_{n}+1=t_{1}$, the goal of traveling salesman problem is to find several permutations that minimize the total distance between cities. It can be represented as $\min f(T)$. The mathematical model can be formulated as Eq.8:

$$
f(T)=\sum_{i=1}^{n-1} d_{t(i) t(i+1)}+d_{t(n) t(1)}
$$

Generally speaking, the traveling salesman problem can be divided into symmetric TSP and asymmetric TSP. In the symmetric TSP, the movement from vertex $i$ to vertex $j$, has the same distance as moving from vertex $j$ to vertex $i$, and it can be expressed as $d_{i j}=d_{j i}$.

Lots of complex problems, such as air route arrangement, printed-circuit board turnhole, human gene sequencing, can be simplified as traveling salesman problems. Traditional methods such as linear programming and integer programming cannot solve the problem effectively. Therefore, how to solve TSP quickly and effectively has important theoretical value and high practical value.

\section{The improved unordered pair bat algorithm}

In this paper, an unordered pair bat algorithm (UPBA) for solving symmetric TSP is proposed. The improved algorithm preserves the basic bat algorithm and corrects it in some aspects. Specifically, the concept of unordered pair is introduced to update the speed and position of bats, which makes it more suitable for solving symmetric discrete traveling salesman problems.

\subsection{The concept of unordered pair}

Unordered pair is a set of two elements. For any two elements $u$ and $v$, set $(u, v)=(v, u)$ is called unordered pair of them. For TSP of $n$ cities, it can be defined that: any two cities $\left(C_{l}\right.$ and $\left.C_{2}\right)$ in the solution space may be connected together to form an ordered pair, called $\left(C_{l}\right.$, $C_{2}$ ). As the distance between any two fixed connected cities is the same in the symmetrical TSP, we define the unordered pair of TSP, which is called $\left(C_{1}, C_{2}\right)=\left(C_{2}, C_{1}\right)$. That is, the unordered pair does not have the concept of sequence.

Take the symmetric TSP of 7 cities (number 1 to 7 ) as an example. The TSP contains 7node and the paths called $x=[5,3,6,7,1,4,2], x_{*}=[5,3,7,6,1,2,4]\left(x_{*}\right.$ is optimal), which are shown in Błąd! Nie można odnaleźć źródła odwołania.. For $x$, it can be regarded as unordered pairs of $(3,5)(3,6)(6,7)(1,7)(1,4)(2,4)$ and $(2,5)$. Here, by default, numbers are arranged from small to big, which is convenient for expression. The unordered pairs between cities mean the connection of cities. We see that the same unordered pairs of $x$ and $x_{*}$ are represented by red color as: $(3,5)(6,7)(2,4)$, and the different unordered pairs are shown by black color as $(3,6)(1,7)(1,4)(2,5)$ and $(3,7)(1,6)(1,2)(4,5)$. The difference between $x$ and $x_{*}$ depends on the number of different unordered pairs. That is, if the different unordered pairs of $x$ are turned into the same as $x_{*}$, then $x$ becomes identical with $x_{*}$, the two paths coincide completely and reach the best solution $x_{*}$. 


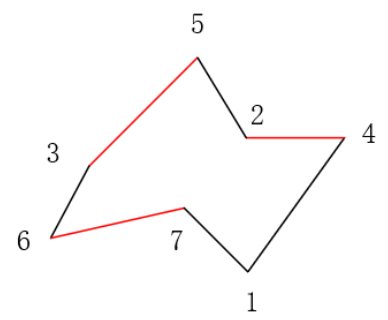

$(x)$

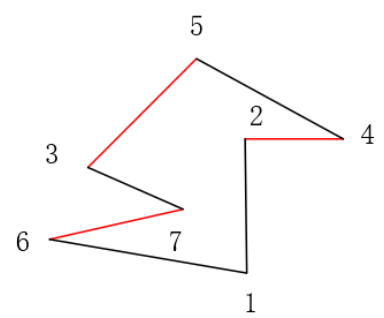

$\left(x_{*}\right)$

Figure 1. An example of TSP with 7-node and feasible solutions

\subsection{Velocity and position updating}

In basic bat algorithm, the position updating is only suitable for solving continuous problems, and it can't be used directly to solve symmetric discrete TSP. Consequently, this paper will search a new set of relationships to update the velocity and position of bats.

The velocity is defined as the distance between the bat's current position and the current best bat, the functional relationship is established to express the updating of velocity of bats by introducing the concept of unordered pair. In a word, the velocity $v_{i}^{t}$ can be described as Eq.9:

$$
v_{i}^{t}=C \operatorname{eil}[\text { rand } * K]
$$

where, $K$ represents the number of different unordered pairs between $x_{i}^{t-1}$ and $x_{*}^{t-1}$. Thus, $v_{i}^{t}$ is a random integer between 1 and $K$.

For symmetric discrete TSP, the position is expressed by exchange process between $v_{i}^{t}$ and $x_{i}^{t-1}$, which is shown by:

$$
x_{i}^{t}=2-o p t\left(v_{i}^{t}, x_{i}^{t-1}\right)
$$

Where, 2-opt $\left(v_{i}^{t}, x_{i}^{t-1}\right)$ presents functional relations for the position of next moment using the velocity and position of the bat. The 2-opt is used to express the random search. Specifically, each bat generates random velocity $v_{i}^{t}$ by Eq.9. Then, the position $x_{i}^{t-1}$ of each bat is transformed by 2-opt for $v_{i}^{t}$ times. Finally, the results of each transformation are compared, and the current best one is selected as the final result.

Here is a simple example in Błąd! Nie można odnaleźć źródła odwołania..

\subsection{Velocity and position updating}

In basic BA, it can be seen that the rate of pulse emission $r_{i}$ increases, while the loudness $A_{i}$ decreases once a bat has found its prey. At the initial stage of iteration, because of the smaller $r_{i}$, each bat has a greater probability of local search. At the same time, larger loudness increases the search area of bats so that they can find better positions. With the iterative generation increasing, the probability of local search is reduced while the global search is increased. Finally, the current best solution is obtained. Therefore, how to optimize $r_{i}$ and $A_{i}$ 
become the focus of the research, which reflects the probability and effectiveness of bat optimization.

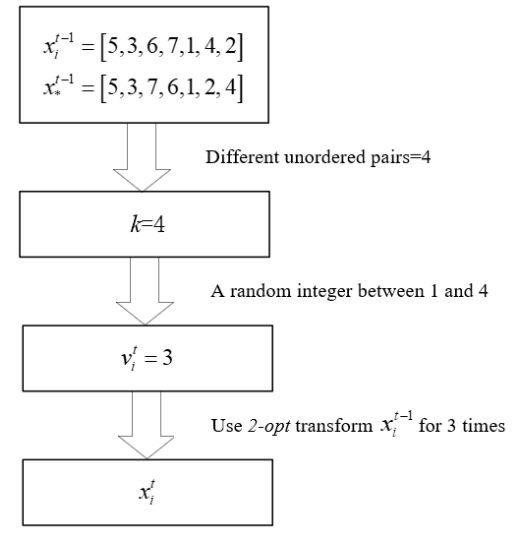

Figure 2. An example of velocity and position updating

It can be considered that the $r_{i}$ and $A_{i}$ are affected by the number of iterations. In basic $\mathrm{BA}$, the loudness is only related to the previous iteration, which may lead to some limitations. Hence, this paper proposes an improved bat algorithm in which the effect of iteration is taken into account. Here, a new definition of $r_{i}$ and $A_{i}$ is proposed to further improve the performance of the algorithm.

$$
\begin{gathered}
A_{i}^{t+1}=A_{i}^{t} * \frac{(\text { iter_max }-t / 8)}{\text { iter_max-iter_min }} \\
r_{i}^{t+1}=\left(1+\exp \left(\frac{8}{\text { iter_max }} *\left(\frac{\text { iter_max }}{2}-\mathrm{t}\right)\right)\right)^{-1}
\end{gathered}
$$

As Eqs.11 and 12, the define of $r_{i}$ and $A_{i}$ are related to the number of iterations. Where, iter_max and iter_min represent the maximum and minimum iterations of the algorithm. By using the above-mentioned equations, each bat has a greater probability of local search at the early stage of iteration, which will accelerate the convergence of the algorithm. At the same time, it effectively prevents the algorithm from falling into a local optimum solution.

\subsection{Unordered pair bat algorithm}

An unordered pair bat algorithm (UPBA) for solving the symmetric TSP is presented below.

Step1: Initialize the size of bat population. The bats generate a set of initial solutions randomly in $D$-dimensional search space. Here, to obtain a better initial solution, a 2-opt method is proposed to optimize the initial position. For TSP with $n$ cities, the initial solution is transformed $n / 2$ times (integer) by 2-opt, and the feasible solution of all is chosen as the current initial position of each individual. Meanwhile, in order to improve the exploration capability of the algorithm, set the initialize pulse emission rate $r_{i} \in[0,0.3]$ and the initialize loudness $A_{i} \in[0.7,1]$.

Step2: Find the current global best location (solution) $x_{*}$ and the initial fitness value. The fitness value is defined as the total distance in symmetric TSP.

Step3: The movement of each bat is given by updating their velocities $v_{i}^{t}$ and positions $x_{i}^{t}$ using Eqs.9 and 10. 
Step4: A random number is generated, if the random number $r a n d>r_{i}$, Select a solution among the best ones, and generate a local solution by using the 2-opt around the selected best solution.

Step5: Evaluate the fitness value.

Step6: A random number is generated, if the random number rand $<A_{i}^{t}$ and $f\left(x_{i}\right)<$ $f\left(x_{*}\right)$, the above solution is accepted and $A_{i}$ and $r_{i}$ are updated by Eqs.11 and 12 .

Step7: Repeat Step3 Step6 until the maximum iterations are achieved.

Step8: Output the best solution, post-process the results and visualization.

\section{Numerical results and performance comparison}

To demonstrate the performance, the proposed UPBA has been tested on 23 symmetric benchmarks of TSP taken from the TSPLIB library. The benchmarks ranging from 14 to 152 cities, and each instance is run for 20 times independently. The UPBA is coded in MATLAB R2016a, and performed on an Intel Core i5 laptop, with 2.9GHz and 8GB RAM.

For the update of pulse emission rate and loudness, to verify that Eqs.11 and 12(UPBA) are better than Eqs.5 and 6 (UPBA1), a contrast test is designed in this paper. At the same time, the comparison between UPBA and UPBA2 is shown below to prove the performance for unordered pair modification.

The comparing results are shown in Tables 3 (the third to eleventh columns). A number shown in bold indicates that the value has reached the optimal solution of the instance. And the second column indicates the optimal solutions obtained by TSPLIB. Best $=$ the best solutions, $S t d=$ the standard deviation of solutions. PDbest represents a relative error when comparing algorithms to the best solution. Table 1 shows the parameter settings of the proposed methods.

$$
\text { PDbest }(\%)=\frac{\text { best solution }- \text { optimal solution }}{\text { optimal solution }} \times 100
$$

As can be seen from the UPBA results, 22 of the current 23 standard problems can find the optimal solutions. In other words, the UPBA can reach the optimal solutions of $95.65 \%$ from the test instances. By contrast, there are 18 and 19 instances can reach the optimal solutions by using the UPBA1 and UPBA2. The percentage of the optimal solutions is $78.26 \%$ and $82.61 \%$, which is obviously weaker than the UPBA. For UPBA, $100.00 \%$ of the values of PDbest $(\%)$ are less than $0.10 \%$, which means that the solutions found by proposed algorithm are close to the optimal solutions after 20 runs. Furthermore, the standard deviations of the UPBA1 and UPBA2are larger than the UPBA. In conclusion, the UPBA is superior to UPBA1 and UPBA2, that is to say, the adjustment of the pulse emission rate and the loudness proposed in this paper is effective, and the proposed unordered pair method is also available.

To evaluate the performance of the above solutions, we compare the UPBA with other algorithms. Tables 3(the twelfth to twentieth columns) show the test results which compare UPBA with GSA-ACS-PSOT algorithm, RABNET-TSP algorithm and Improved BA. The optimal solutions obtained by these algorithms are shown in bold. Tables 1 and Tables 2 show the parameter settings of the proposed methods. 
Table 1. Parametrization of the UPBA, UPBA1, UPBA2 and Improved BA for the TSP

\begin{tabular}{|l|l|l|l|l|}
\hline & UPBA & UPBA1 & UPBA2 & Improved BA \\
\hline Parameter & Value & Value & Value & Value \\
\hline Population size & 50 & 50 & 50 & 50 \\
\hline Velocity $v_{i}^{t}$ & Unordered pair & Unordered pair & Random & Hamming Distance \\
\hline Movement functions & 2-opt\&A\&r & 2 -opt & 2-opt\&A\&r & 2-opt\&3-opt \\
\hline Initial $A_{i}$ & {$[0.7-1.0]$} & {$[0.7-1.0]$} & {$[0.7-1.0]$} & {$[0.7,1.0]$} \\
\hline Initial $r_{i}$ & {$[0.0-0.3]$} & {$[0.0-0.3]$} & {$[0.0-0.3]$} & {$[0.0-0.4]$} \\
\hline$\alpha$ and $\gamma$ & 0.9 & 0.9 & 0.9 & 0.98 \\
\hline
\end{tabular}

Table 2. Parametrization of the GSA-ACS-PSOT and RABNET-TSP for the TSP

\begin{tabular}{|l|l|l|l|}
\hline & GSA-ACS-PSOT & & RABNET-TSP \\
\hline Parameter & Value & Parameter & Value \\
\hline Population size & $\begin{array}{l}4 \text { groups, 30 ants } \\
\text { each group }\end{array}$ & Initial value for $\sigma$ & $\sigma(0)=16$ \\
\hline Pheromone evaporation parameter & 0.1 & Initial learning rate & $a(0)=0.1$ \\
\hline Parameter for crossover strategy & 0.33 & Cooperation threshold & $k=0.01$ \\
\hline The number of GA generations & 100 & learning rate & 0.6 \\
\hline Crossover rate & 1 & Convergence threshold & $\lambda=0.01$ *smallest distance \\
\hline Route mutation rate & 0.3 & Cloning threshold & $\varepsilon=0.2 *$ smallest distance \\
\hline Pheromone mutation rate & 0.2 & - & - \\
\hline $\begin{array}{l}\text { Cycles for pheromone } \\
\text { communication }\end{array}$ & 30 & - & - \\
\hline
\end{tabular}

According to the twelfth to seventeenth columns, among the selected 13 tested instances, the UPBA can acquire the optimal solutions for 13 instances, while the GSA-ACS-PSOT algorithm and RABNET-TSP algorithm find the optimal solutions for 9 and 2 instances respectively. Moreover, the average standard deviations of the three algorithms are 60.54 , 79.95 and 123.92. The smaller the average standard deviation, the better the stability of the instances results. According to the eighteenth to twentieth columns, for the 16 tested instances, the UPBA acquires the optimal solutions for 15 instances, while the Improved BA finds the optimal solutions for only 12 instances. Besides, the average standard deviations of the two algorithms are 102.16 and 172.15. In summary, the UPBA has maximum optimal solutions instances and minimum average standard deviations, it gets better solutions among the above algorithms.

Błąd! Nie można odnaleźć źródła odwolania. shows the standard deviation of the algorithms. Figures (a) to (d) show the comparison of the average solution and standard deviation of the algorithms under different instances. It can be seen that the UPBA algorithm is always in a better solution. Błąd! Nie można odnaleźć źródła odwołania. shows the relative error between the best solution and the TSPLIB optimum solution obtained by each algorithm. As can be seen from the figure, the relative error of UPBA is the smallest, which is indicated in red at the bottom of the plot. That is to say, the UPBA is closer to the optimal solution and has the best performance. Błąd! Nie można odnaleźć źródła odwołania. expresses the different running times of the UPBA, RABNET-TSP and Improved BA algorithms. As can be seen from the figure, the running time of the UPBA algorithm is also excellent. 


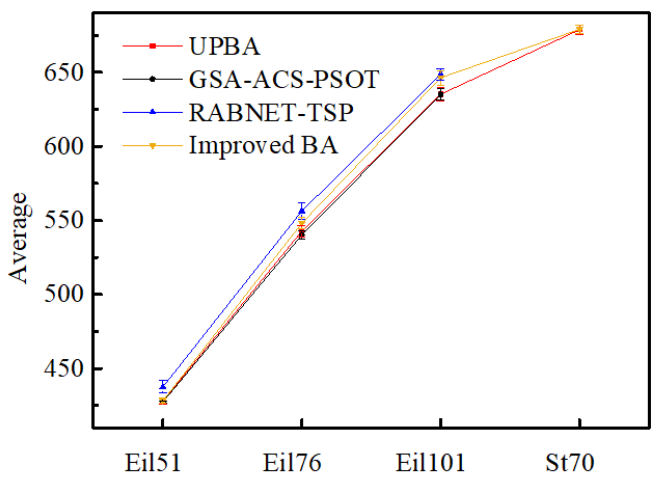

(a)

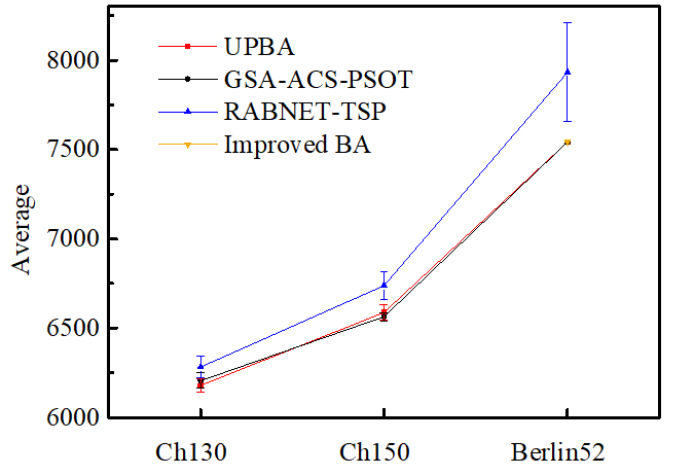

(b)

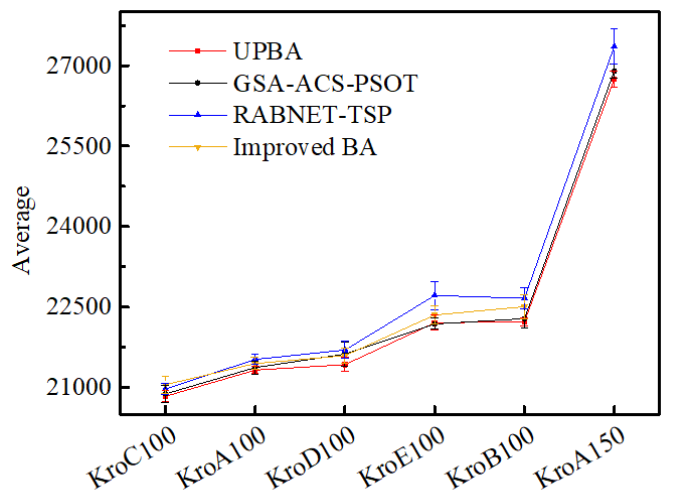

(c) 


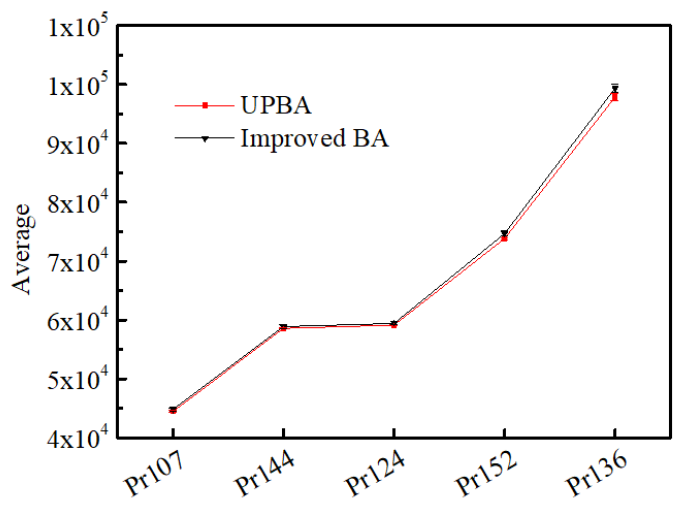

(d)

Figure 3. Average solution and standard deviation of algorithms

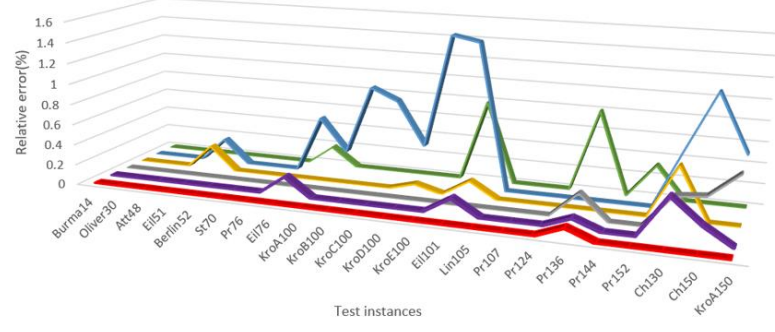

-

Figure 4. Relative error of algorithms

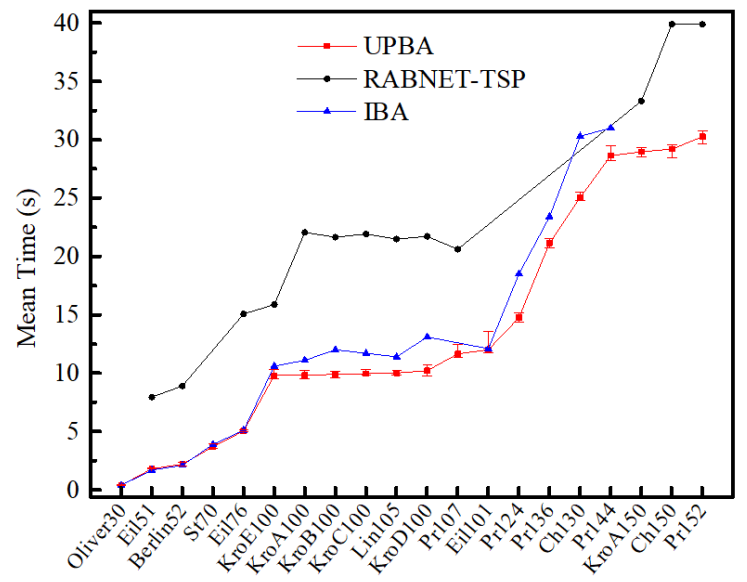

Figure 5. Running time of algorithms 


\section{Conclusion}

This paper has proposed an improved bat algorithm to solve the symmetric TSP. In the course of improvement, the concept of unordered pair is introduced to update the velocity and position of bats. Moreover, a new definition of $r_{i}$ and $A_{i}$ is proposed. Finally, an unordered pair bat algorithm is presented. The algorithm has been evaluated through solving 23 instances of the symmetric TSP, and its performance has been compared with GSA-ACSPSOT algorithm, RABNET-TSP algorithm and the Improved BA. Among the selected tested instances from cities 51 to 150 , the UPBA acquires the optimal solutions for all 13 instances, while the GSA-ACS-PSOT and RABNET-TSP algorithms for 9 and 2 instances respectively. Similarly, from cities 30 to 152, the UPBA finds the optimal solutions for 15 instances, while the Improved BA for only 12 instances. Moreover, the UPBA has minimum average standard deviations. So, the results show that the UPBA proposed in this paper is superior to all of the above algorithms. For future research, the UPBA can be considered to solve larger-scale TSP. And the algorithm can also be considered to solve other combinatorial optimization problems, such as vehicle routing problems, bin packing problems, clustering problems, and so on.

\section{Acknowledgment}

This research is supported by the National Natural Science Foundation of China (grant number 51274043). 


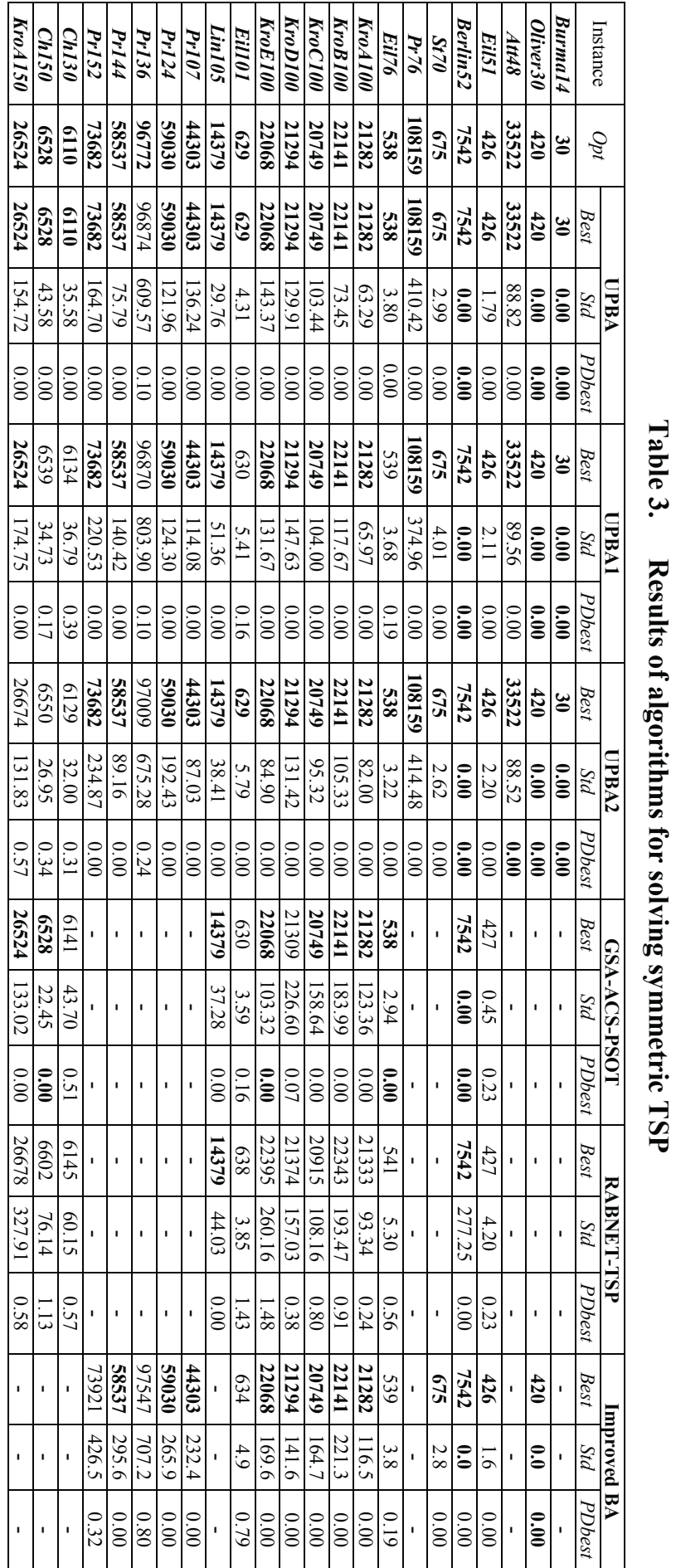




\section{References}

[1] Abdel-Raouf O, Abdel-Baset M, El-Henawy I., An improved chaotic bat algorithm for solving integer programming problems, International Journal of Modern Education and Computer Science, 6, 8, 2014, 18.

[2] Afrabandpey H, Ghaffari M, Mirzaei A, et al., A novel bat algorithm based on chaos for optimization tasks, 2014 Iranian Conference on Intelligent Systems (ICIS), IEEE, 2014, $1-6$.

[3] Al-Sorori, Wedad, A., Mohsen, and Walid Aljoby ßer., An Improved Hybrid Bat Algorithm for Traveling Salesman Problem, International Conference on Bio-Inspired Computing: Theories and Applications, Springer, Singapore, 2016: 504-511.

[4] Chen S M, Chien C Y., Solving the traveling salesman problem based on the genetic simulated annealing ant colony system with particle swarm optimization techniques, Expert Systems with Applications, 38, 12, 2011, 14439-14450.

[5] Cheng H, Yang S, Cao J, Dynamic genetic algorithms for the dynamic load balanced clustering problem in mobile ad hoc networks, Pergamon Press, Inc, 2013.

[6] Crişan G C, Pintea C M, Palade V., Emergency management using geographic information systems: application to the first romanian traveling salesman problem instance, Knowledge and Information Systems, 50, 1, 2017, 265-285.

[7] Dorigo M, Di Caro G, Ant colony optimization: a new meta-heuristic, Proceedings of the 1999 congress on evolutionary computation-CEC99 (Cat. No. 99TH8406), IEEE, 2,1999, 1470-1477.

[8] Eberhart R, Kennedy J, Particle swarm optimization, Proceedings of the IEEE international conference on neural networks, 4, 1995, 1942-1948.

[9] Fischer A, Fischer F, Jäger G, et al., Exact algorithms and heuristics for the quadratic traveling salesman problem with an application in bioinformatics, Discrete Applied Mathematics, 166, 2014, 97-114.

[10]Fister Jr I, Fister D, Yang X S., A hybrid bat algorithm, arXiv preprint arXiv, 2013,1303.6310.

[11] Gandomi A H, Yang X S, Alavi A H, Cuckoo search algorithm: a metaheuristic approach to solve structural optimization problems, Engineering with computers, 29(1), 2013, 17 35 .

[12] Gandomi A H, Yang X S., Chaotic bat algorithm, Journal of Computational Science, 5(2), 2014, 224-232.

[13] Glover F, Tabu search—part I, ORSA Journal on computing, 1(3), 1989, 190-206.

[14]He X, Ding W J, Yang X S., Bat algorithm based on simulated annealing and Gaussian perturbations, Neural Computing and Applications, 25, 2, 2014, 459-468. 
[15]Hoffman K L, Padberg M, Rinaldi G., Traveling salesman problem, Encyclopedia of operations research and management science, Springer US, 2013, 1573-1578.

[16]Lin Y, Bian Z, Liu X., Developing a dynamic neighborhood structure for an adaptive hybrid simulated annealing-tabu search algorithm to solve the symmetrical traveling salesman problem, Applied Soft Computing, 49, 2016, 937-952.

[17] Mahi M, Baykan Ö K, Kodaz H., A new hybrid method based on particle swarm optimization, ant colony optimization and 3-opt algorithms for traveling salesman problem, Applied Soft Computing, 30, 2015, 484-490.

[18] Marichelvam M K, Prabaharam T., A bat algorithm for realistic hybrid flowshop scheduling problems to minimize makespan and mean flow time, ICTACT Journal on Soft Computing, 3, 1, 2012, 428-433.

[19] Marichelvam M K, Prabaharan T, Yang X S, et al., Solving hybrid flow shop scheduling problems using bat algorithm, International Journal of Logistics Economics and Globalisation, 5, 1, 2013, 15-29.

[20] Masutti T A S, de Castro L N., A self-organizing neural network using ideas from the immune system to solve the traveling salesman problem, Information Sciences, 179, 10, 2009, 1454-1468.

[21] Mirjalili S, Mirjalili S M, Yang X S., Binary bat algorithm, Neural Computing and Applications, 25(3-4), 2014, 663-681.

[22] Osaba E, Yang X S, Diaz F, et al., An improved discrete bat algorithm for symmetric and asymmetric Traveling Salesman Problems, Engineering Applications of Artificial Intelligence, 48, 2006, 59-71.

[23] Ouaarab A, Ahiod B, Yang X S., Discrete cuckoo search algorithm for the travelling salesman problem, Neural Computing and Applications, 24, 7-8, 2014, 1659-1669.

[24]Pedro O, Saldanha R, Camargo R., A tabu search approach for the prize collecting traveling salesman problem, Electronic Notes in Discrete Mathematics, 41, 2013, 261 268.

[25]Plateau G, Nagih., A 0-1 Knapsack Problems. Paradigms of Combinatorial Optimization, 2nd Edition, 2010, 215-242.

[26] Quiroz-Castellanos M, Cruz-Reyes L, Torres-Jimenez J et al., A grouping genetic algorithm with controlled gene transmission for the bin packing problem, Computers \& Operations Research, 55, 2015, 52-64.

[27] Saji Y, Riffi M E., A novel discrete bat algorithm for solving the travelling salesman problem, Neural Computing and Applications, 2015, 1-14.

[28]Toth, Paolo, and Daniele Vigo, eds., Vehicle routing: problems, methods, and applications, Society for Industrial and Applied Mathematics, 2014.

[29] Van Laarhoven P J M, Aarts E H L, Simulated annealing, Simulated annealing: Theory and applications, Springer, Dordrecht, 1987, 7-15. 
[30] Wang J, Ersoy O K, He M, et al., Multi-offspring genetic algorithm and its application to the traveling salesman problem, Applied Soft Computing, 43, 2016, 415-423.

[31] Whitley D, A genetic algorithm tutorial, Statistics and computing, 4(2), 1994, 65-85.

[32] Yang X S., A new metaheuristic bat-inspired algorithm, Nature inspired cooperative strategies for optimization (NICSO 2010), Springer Berlin Heidelberg, 2010, 65-74.

[33]Zhou Y, Xie J, Li L, et al., Cloud model bat algorithm, The Scientific World Journal, 2014.

Received 07.04.2021, Accepted 17.12.2021 